einer Partei beginnt immer auf der rechten oberen Seite im Stimmzettelheft (um das Problem des Listenplatzes $31 \mathrm{zu}$ beseitigen) und beschränkt sich im Idealfall auf eine Seite (um nicht die Kandidaten auf der Rückseite zu benachteiligen). Damit dies graphisch machbar ist, beschränkt man sich auf eine Namensnennung und lässt Jahrgangs- wie Berufsbezeichnung weg (auch um ein „heiteres Beruferaten“ der Wähler zu vermeiden). Der rote Stimmzettel (Wahlkreisliste) könnte bis auf die oben erwähnte Kürzung so bleiben und auch weiterhin Orts-, Jahrgangs- und Berufsangabe sowie - als Neuerung - ein Foto enthalten.

(2) Eine mittelgroße Lösung wäre die Rückkehr zu geschlossenen Landeslisten, die gegenüber „Mehr Demokratie“ jedoch eine hohe Überzeugungskraft und somit auch Einigkeit der Parteien verlangen würde. Da unklar ist, ob die CDU nach den für sie wenig erbaulichen Erfahrungen der Vergangenheit der SPD die Hand reichen wird, könnte eine solche Lösung am erfolgversprechendsten von einer Linkskoalition aus SPD, GAL und Linkspartei, die in der Bürgerschaft über eine Zweidrittelmehrheit verfügt, angestrebt werden. Der entscheidende Vorzug geschlossener Landeslisten besteht darin, dass die Einfachheit und Verständlichkeit des Wahlrechts erheblich befördert würde. Bliebe man beim Mehrstimmenwahlrecht, würden die fünf Landesstimmen (Zweitstimmenpaket) der Parteienwahl vorbehalten bleiben, die fünf Wahlkreisstimmen (Erststimmenpaket) der Personenwahl im Wahlkreis. Die Möglichkeit des Panaschierens und Kumulierens könnte erhalten werden - auf Landesebene zwischen den Gesamtlisten der Parteien, auf Wahlkreisebene beschränkt auf die Kandidaten. Damit könnten Parteien, Kandidaten und Wähler gut leben: Die Parteien behielten den Einfluss über das (nun wieder vergrößerte) Listensegment der Abgeordneten, die Kandidaten derselben Partei wären froh, nur im Wahlkreis miteinander konkurrieren zu müssen; und die Wähler hätten eine deutlich bessere Übersicht zurückgewonnen: Zumindest der Landeslistenstimmzettel könnte wieder ein Zettel sein (und kein Heft); der Wähler könnte seine Aufmerksamkeit auf die - einigermaßen überschaubare Anzahl von - Kandidaten im Wahlkreis vor Ort konzentrieren; er wüsste wieder etwas besser, welche Wirkungen das eigene Stimmverhalten auf das Wahlergebnis hat; und er könnte sogar - ganz unabhängig von der Personenwahl im Wahlkreis - allein mit seinem Zweitstimmenpaket tatsächliche Koalitionspräferenzen ausdrücken. Nicht ganz unberechtigt erschiene in diesem Fall die Hoffnung, dass die Zahl ungültig oder gar nicht wählender Bürger wieder zurückgehen würde. Der Akzeptanz der parlamentarischen Demokratie in Hamburg könnte dies nur gut tun.

\title{
Die Wahl zur Hamburger Bürgerschaft vom 20. Februar 2011: Ehemalige „Hamburg-Partei““ erobert absolute Mehrheit zurück
}

\section{Patrick Horst}

Hamburg machte zwar im Februar den Auftakt zum Superwahljahr 2011 mit seinen sieben Landtagswahlen, hatte aber nur geringe bundespolitische Bedeutung: Den Verlust der drei Bundesratsstimmen Hamburgs konnte die schwarz-gelbe Koalition in Berlin verschmerzen, denn sie hatte bereits im Juli 2010 nach der Landtagswahl in Nordrhein-Westfalen die Mehrheit in der zweiten Kammer verloren. Für Hamburg bedeutete dies, dass eine bundes- 
politische Überformung der Wahl wie schon bei vergangenen Bürgerschaftswahlen ${ }^{1}$ nicht gegeben war und landespolitische Motive eindeutig im Vordergrund standen. ${ }^{2}$

\section{Die Ausgangslage}

Der Stadtstaat Hamburg hatte im Mai 2008 auch bundespolitisch einiges Aufsehen erregt, als CDU und Grüne (GAL) nach einem 73 Tage dauernden Regierungsbildungsprozess die erste schwarz-grüne Koalition in einem deutschen Bundesland bildeten. ${ }^{3}$ Auch wenn die Koalitionspartner betonten, kein Modell für Berlin sein zu wollen, stellte das Bündnis faktisch einen ersten Probelauf für diese neue Farbenkombination dar und wurde folglich auch in der Bundeshauptstadt mit Interesse beobachtet. Darüber hinaus war es ein interessantes Fallbeispiel für eine ideologisch heterogene Koalition, die sich nach den Prämissen einer spieltheoretisch argumentierenden Forschung gar nicht hätte bilden dürfen oder aber zumindest unter der Erwartung ihres baldigen Scheiterns stand. Die lange Zeit vorherrschende Annahme, dass ideologisch unverbundene Koalitionen instabiler seien als verbundene wurde in der internationalen Koalitionsforschung jedoch jüngst empirisch widerlegt; sie haben sich sogar überraschenderweise als stabiler herausgestellt. Der Grund wird darin vermutet, dass die Partner ideologisch unverbundener Koalitionen nicht um dieselben Segmente der Wählerschaft konkurrieren und sich auf ihren jeweiligen Kompetenzfeldern relativ ungestört betätigen können, was diese Bündnisse weniger konfliktanfällig mache. ${ }^{4}$ Die Hamburger Partner schlossen sich dieser Sichtweise an und vermarkteten sich selbstbewusst als eine „Ergänzungskoalition“, die ihre jeweiligen Stärken addieren wollte. ${ }^{5}$ So trugen die Grünen das CDU-Projekt der „wachsenden Stadt“ (Elbvertiefung, Elbphilharmonie, große Straßenverkehrsprojekte), den Bau des Kohlekraftwerks Moorburg und die Beibehaltung der (auf Wunsch der Grünen immerhin reduzierten) Studiengebühren mit, während die CDU im Gegenzug der Einführung einer sechsjährigen Primarschule, eines Straßenbahnprojekts („Stadtbahn“) und eines städtischen Radverleihsystems zustimmte. ${ }^{6}$

Knapp zwei Jahre lang ging die Rechnung auf: CDU und Grüne bearbeiteten jeweils ihre Kompetenzfelder; die Atmosphäre auf Senatsebene war laut Presseberichten von Harmonie

1 Zu den letzten drei Bürgerschaftswahlen vgl. Patrick Horst, Die Hamburger Bürgerschaftswahl vom 23. September 2001: Schillerndem „Bürger-Block“ gelingt der Machtwechsel, in: ZParl, 33. Jg. (2002), H. 1, S. 43 - 63; ders., Die Neuwahl zur Hamburger Bürgerschaft vom 29. Februar 2004: Die Hanseaten und Angela Merkel finden einen politischen Hoffnungsträger, in: ZParl, 35. Jg. (2004), H. 2, S. 252 - 270; ders., Die Wahl zur Hamburger Bürgerschaft vom 24. Februar 2008: Wahlsieger Ole von Beust bildet die erste schwarz-grüne Koalition auf Landesebene, in: ZParl, 39. Jg. (2008), H. 3, S. $509-528$.

2 Laut Forschungsgruppe Wahlen, Wahl in Hamburg. Eine Analyse der Bürgerschaftswahl vom 20. Februar 2011, Mannheim, Februar 2011, S. 14, war dies für knapp 80 Prozent der potenziellen Wähler der Fall.

3 Vgl. Patrick Horst, Wahl 2008, a.a.O. (Fn. 1), S. 526.

4 Dazu Thomas Saalfeld, Parteiensystem und Kabinettsstabilität in Westeuropa 1945-1999, in: Oskar Niedermayer (Hrsg.), Die Parteiensysteme Westeuropas, Wiesbaden 2006, S. 492 - 493; ders., Die Stabilität von Koalitionsregierungen im europäischen Vergleich: Empirische Befunde und institutionelle Erklärungsansätze, in: Klemens H. Schreck / Markus Söldner (Hrsg.), Analyse demokratischer Regierungssysteme, Wiesbaden 2010, S. 520 - 521.

5 Siehe die Präambel des Koalitionsvertrags vom 17. April 2008, S. 4.

6 Vgl. Patrick Horst, Wahl 2008, a.a.O. (Fn. 1), S. 525. 
und Konfliktfreiheit geprägt. Vor allem im Spitzenquartett der Koalition, gebildet von Bürgermeister Ole von Beust, Finanzsenator Michael Freytag (beide CDU), Bildungssenatorin Christa Goetsch und Stadtentwicklungssenatorin Anja Hajduk (beide GAL), stimmte angeblich die politische Chemie. Konflikte gab es wenn überhaupt innerhalb der Parteien zwischen Führung und Mitgliedschaft, die bei der CDU wie bei der GAL der Koalition deutlich kritischer gegenüberstand als ihr Führungspersonal. Noch im Februar 2009 zeigten sich 41 Prozent der Hamburger mit der Arbeit des Senats zufrieden. Erst im Winter 2009/10 geriet die Koalition dann im Strudel der Finanzkrise und auf dem Eis der nicht gestreuten Hamburger Verkehrswege ins Schleudern; im Februar 2010 äußerten sich bereits sieben von zehn Hamburgern unzufrieden über die Arbeit des Senats. ${ }^{7}$ Der Nimbus der tragenden Koalitionssäule Hajduk war ebenso zerbrochen wie derjenige des CDU-Parteivorsitzenden Freytag, der im März 2010 im Gefolge zahlreicher Affären bei der HSH Nordbank und eines stark angestiegenen Haushaltsdefizits von allen Partei- und Regierungsämtern zurücktrat. Seitdem hielten sich hartnäckig die Mediengerüchte, dass auch Bürgermeister von Beust von Amtsmüdigkeit heimgesucht werde. Diese wurden durch eine innerparteiliche Machtarrondierung der beiden damals „starken Männer“ in Hamburgs CDU genährt, die schon eine Nachfolgeregelung für das Bürgermeisteramt beinhaltete: Innensenator Christoph Ahlhaus überließ Fraktionschef Frank-Thorsten Schira den Parteivorsitz; Schira verzichtete im Gegenzug zugunsten von Ahlhaus auf eine etwaige Bürgermeisterkandidatur 2012. ${ }^{8}$ Als die Hamburger Stimmbürger dann am 18. Juli 2010 in einem Volksentscheid die Einführung der Primarschule ablehnten, wurden die Ahnungen zur Gewissheit: Der Bürgermeister, zusammen mit Bildungssenatorin Goetsch entschiedener Befürworter der Schulreform, erklärte seinen Rücktritt zum 25. August. Mit ihm gingen der Chef der Senatskanzlei Volkmar Schön, Kultursenatorin Karin von Welck und Wirtschaftssenator Axel Gedaschko. ${ }^{9}$

In Hamburg hatten die Grünen besonders viel Vertrauen in den Bürgermeister gesetzt, der aus ihrer Sicht den Garanten für den liberalen Kurs der Hamburger Union und für die Koalition insgesamt darstellte. Der Rücktritt von Beusts war für die Grünen ein Schock. Für die Koalitionsforschung ist der Rücktritt eines Regierungschefs immer ein „kritisches Ereignis“, das umso eher zum Bruch des Bündnisses führt, je näher der Wahltermin rückt. ${ }^{10} \mathrm{Mit}$ der Niederlage der Koalition im Volksentscheid gegen die Primarschulreform war zeitgleich ein zweiter externer Schock hinzugekommen, der den Grünen ihr wichtigstes Vorhaben aus der Hand schlug. Dem neuen Bürgermeister der CDU, Christoph Ahlhaus, der über ein konservativeres Profil und eine in den Augen der Grünen weit geringere politische Statur als sein Vorgänger verfügte, konnte es nur kurze Zeit gelingen, die GAL zur Fortsetzung der Koalition zu bewegen. Ahlhaus' Personalentscheidungen in Gestalt des neuen Wirtschaftssenators Ian Karan und des Kultursenators Reinhard Stuth erwiesen sich als wenig glücklich $^{11}$; sein Festhalten am angeschlagenen Finanzsenator Carsten Frigge, gegen den im Rah-

7 Vgl. Infratest dimap, Bürgerschaftswahl Hamburg 2011. Eine Analyse der Wahl vom 20. Februar 2011 (WahlREPORT Hamburg 2011), Berlin, April 2011, S. 23.

8 Dazu „Beust-Nachfolge: Innensenator Ahlhaus neuer Kronprinz“, Hamburger Abendblatt, 3. März 2010, S. 1.

9 Vgl. „Beust tritt zurück - Schulreform scheitert“, faz.net, 18. Juli 2010, „Wirtschaftssenator Axel Gedaschko geht nach Berlin“, abendblatt.de, 27. Juli 2010 (Abruf am 14. Juli 2011).

10 Siehe Thomas Saalfeld, Stabilität, a.a.O. (Fn. 4), S. 514 ff.

11 Vgl. die vernichtende Kritik von Uwe Bahnsen, Die großen Fehler der Hamburger CDU, welt.de, 29. November 2010 (Abruf am 15. Januar 2011). 
men der rheinland-pfälzischen CDU-Parteispendenaffäre staatsanwaltlich ermittelt wurde, entwickelte sich zur Belastungsprobe der Koalition. Weil den Grünen zudem nicht verborgen geblieben war, dass ihre Werte in den Hamburger Wahlumfragen, traditionell eine Grünen-Hochburg, deutlich hinter dem deutschlandweiten Höhenflug zurückblieben ${ }^{12}$, versprachen sie sich von einer Aufkündigung der Koalition mehr Vor- als Nachteile. Als Finanzsenator Frigge am 24. November seinen Rücktritt anbot, ergriffen die Grünen vier Tage später dankbar die Gelegenheit, die Koalition aufzukündigen. ${ }^{13}$ Damit war der Weg frei zur Selbstauflösung der Hamburger Bürgerschaft. ${ }^{14}$

Nach dem Auflösungsbeschluss legte der Senat den Wahltermin innerhalb der vorgesehenen zehnwöchigen Frist auf den 20. Februar 2011 fest. Damit blieb den Parteien kaum Zeit zur Führung eines intensiven Wahlkampfes. Der Bedarf danach schien auch bei allen gering, denn die demoskopische Ausgangslage war so eindeutig wie selten zuvor: Zwei Drittel der Wahlberechtigten sprachen sich im Dezember 2010 für einen politischen Wechsel in der Hansestadt aus und wünschten sich eine Regierung unter Führung der SPD; unter den Grünen-Anhängern waren es neun von zehn. Bei der Sonntagsfrage erzielten die Sozialdemokraten im Vergleich zum Februar 2010, als sie noch gleichauf mit der CDU bei 31 Prozent gelegen hatten, einen Zuwachs um 12 Punkte auf 43 Prozent, während die regierende CDU auf 22 Prozent absank und die übrigen Parteien (die Linke mit sieben, die FDP mit vier Prozent) um den Einzug in die Bürgerschaft bangten. Anders als die Christdemokraten profitierten die Grünen vom Ende der Koalition. Ihre Umfragewerte schnellten auf 19 Prozent hoch, zusammen lag Rot-Grün damit bei 62 Prozent. Der SPD-Landesvorsitzende Olaf Scholz distanzierte CDU-Bürgermeister Christoph Ahlhaus mit mehr als 40 Punkten (66 zu 19 Prozent) in der „Direktwahlfrage“. Schwarz-Grün gehörte in Hamburg definitiv der Vergangenheit an, vor der Tür stand nach knapp zehn Jahren in der Opposition die Rückkehr der „Hamburg-Partei“ SPD an die Macht. Ob sie dazu einen Koalitionspartner benötigen würde, war die spannende Frage. ${ }^{15}$

\section{Der Wahlkampf}

Mit der Aufkündigung der Koalition durch die GAL war der Wahlkampf ausgebrochen: Noch am selben Tage erklärte Scholz nach einer Sitzung des Parteivorstands seine Kandidatur zum Bürgermeister. ${ }^{16}$ Scholz, der den SPD-Landesvorsitz erst nach der verlorenen Bundestagswahl 2009 übernommen hatte, war in Partei und Fraktion unumstritten, nachdem er in relativ kurzer Zeit die tief zerstrittene Partei befriedet hatte. Mit Wahlprognosen von

12 In der Sonntagsfrage zur Hamburger Bürgerschaftswahl pendelten die Grünen von April bis November 2010 zwischen Werten von zehn und zwölf Prozent, deutschlandweit dagegen zwischen 16 und 23 Prozent. Vgl. http://www.wahlrecht.de/umfragen/landtage/hamburg.htm und http:// www.infratest-dimap.de/umfragen-analysen/bundesweit/sonntagsfrage (Abruf am 30. Juli 2011).

13 Vgl. „GAL sprengt Koalition - Neuwahl im Februar - Scholz (SPD) tritt an“, Hamburger Abendblatt, 29. November 2010, S. 1, S. 2, S. 7 - 10.

14 Siehe Hamburgische Bürgerschaft, Plenarprotokoll 19/68 vom 15. Dezember 2010, S. 4223 4242.

15 Vgl. Infratest dimap, LänderTREND Hamburg, Dezember 2010, Berlin.

16 Siehe „Olaf Scholz will Bürgermeister werden“, Hamburger Abendblatt, 29. November 2010, S. 7. 
deutlich über 40 Prozent im Rücken, konnte er siegesgewiss sein. In einem großen Zeitungsinterview ein Tag nach dem Zusammenbruch der Koalition erklärte er eine Mehrheit für Rot-Grün zum Wahlkampfziel; eine Zusammenarbeit mit der Linken schloss er aus. Erste inhaltliche Schwerpunkte seines Wahlkampfs kündigte er auch bereits an: Die vom Vorgängersenat beschlossene Erhöhung der Kita-Gebühren wollte er wieder zurücknehmen, den Bau der Stadtbahn wahrscheinlich auf Eis legen und den Haushalt konsolidieren. Außerdem wollte er der "Sehnsucht" der Hamburger nach „Seriosität, Verlässlichkeit und mehr Pragmatismus" als Bürgermeister nachkommen - womit er seinen späteren zentralen Wahlkampfslogan inhaltlich vorwegnahm. ${ }^{17}$ Von der SPD unter Zugzwang gesetzt, nominierten auch die restlichen drei Rathausparteien ihre Spitzenkandidaten bereits am 29. November: Für die CDU trat Bürgermeister Ahlhaus erneut an; bei der GAL verzichtete die bisherige Zweite Bürgermeisterin Goetsch zugunsten von Hajduk; die Linke nominierte wieder ihre Fraktionsvorsitzende Dora Heyenn. ${ }^{18}$ Die seit 2004 nicht mehr in der Bürgerschaft vertretene FDP präsentierte am 14. Dezember die erst 35-jährige, politisch bisher kaum in Erscheinung getretene, dafür aber sehr adrette Katja Suding als Spitzenkandidatin. ${ }^{19}$

Der Wahlkampf der CDU war von Beginn an davon geprägt, dass die Partei bei einem Wählerrückhalt von nur noch etwas mehr als 20 Prozent über keine realistische Machtoption verfügte. Bürgermeister Ahlhaus konnte sich noch so sehr mühen, den Grünen „Flucht vor der Verantwortung" vorzuwerfen und seiner menschlichen Enttäuschung über ihren Koalitionsbruch Ausdruck zu verleihen ${ }^{20}$ : In der Wahrnehmung der Hamburger blieb der Eindruck haften, dass vornehmlich die CDU mit ihren fünf Senatoren-Rücktritten seit März die Koalition im Stich gelassen hatte. Ahlhaus sah sich von allen potenziellen Bündnispartnern verlassen und suchte sein Heil in der Abgrenzung vom ehemaligen Koalitionspartner: Gleich am Morgen des 29. November händigte er den grünen Senatorinnen Goetsch, Hajduk und Justizsenator Till Steffen die Entlassungsurkunden aus. Einen Tag später stoppte er die Planungen zum Bau der Stadtbahn ${ }^{21}$, noch einmal zwei Tage später verkündete er das Aus für die von der GAL betriebene Reform des Schulnotensystems. In einem großem Zeitungsinterview lastete er die Probleme der Senatspolitik in der Vergangenheit vor allem Stadtentwicklungssenatorin Hajduk an: Weil „Ideologie über die Sorgen der Menschen gestellt" worden sei, hätte es mit der Schneeräumung im Winter, der BaustellenKoordinierung, der Beseitigung der Schlaglöcher auf den Straßen und mit dem Wohnungsbau gehapert. ${ }^{22}$ Auch von seinem Vorgänger von Beust grenzte sich Ablhaus ab: Ihm warf er vor, den CDU-Markenkern in der Schulpolitik aufgegeben zu haben. ${ }^{23}$ Da passte es ins Konzept, dass er nur wenig später den Initiator der Volksinitiative „Wir wollen lernen“, Walter Scheuerl, als parteilosen Kandidaten auf der CDU-Liste präsentieren konnte. Scheuerl hatte kurze Zeit überlegt, ob er mit einer eigenen Partei zur Bürgerschaftswahl antreten

17 Zitiert in: „Wir werden die Kita-Gebühren wieder senken“, ebenda, 30. November 2010, S. 9.

18 Vgl. „Scholz schließt Rot-Rot-Grün aus“, ebenda, 30. November 2010, S. 1.

19 Die Medien belustigten sich über „FDP’s Next Topmodel“ und den „Silvana-Effekt“, wonach eine attraktive Spitzenkandidatin mit höheren Wähleranteilen rechnen könne als ein unbeliebter Parteichef - nachweisbar erstmals bei der Europawahlkampagne der FDP 2004 mit Silvana KochMehrin an der Spitze. Vgl. Peter Dausend, Der Silvana-Effekt, Die Zeit, 13. Januar 2011, S. 8.

20 Zitiert in: „Ein Anruf erschüttert die CDU“, Hamburger Abendblatt, 29. November 2010, S. 9.

21 Vgl. „Ahlhaus stoppt die Hamburger Stadtbahn“, ebenda, 1. Dezember 2010, S. 1.

22 Zitiert in: „Jetzt heißt es kämpfen“, ebenda, 3. Dezember 2010, S. 11.

23 Vgl. „Ahlhaus geht auf Distanz zu Beust“, ebenda, 8. Dezember 2010, S. 8. 
würde, sah sich in der knappen Zeit bis zur Wahl jedoch vor zu große Organisations- und Personalprobleme gestellt. Nachdem er von CDU und FDP Angebote erhalten hatte, auf ihrer Liste zu kandidieren, entschied der Blankeneser Rechtsanwalt sich für den sicheren Listenplatz fünf der CDU. ${ }^{24}$ Dass Ahlhaus vor allem auf die Schärfung des konservativen Profils und die Mobilisierung der Stammwählerschaft setzte, machte er nach Weihnachten noch einmal deutlich, als er zusammen mit Innensenator Heino Vahldieck für schärfere Sicherheitsgesetze und die Vorratsdatenspeicherung eintrat. ${ }^{25}$

Neben OlafScholz, Christoph Ahlhaus und Walter Scheuerl, der noch mit seiner Weigerung, zusammen mit Ole von Beust Wahlkampf zu führen, Aufruhr in der CDU auslöste ${ }^{26}$, machten im relativ ereignisarmen Dezember nur noch die Grünen ein wenig von sich reden: Auf ihrer Landesmitgliederversammlung am 13. Dezember zog die GAL entgegen ihren basisdemokratischen Prinzipien die Nominierung der Spitzenkandidatin vor die Diskussion des Wahlprogramms, die erst für den Januar vorgesehen war. Die mit nur einer Gegenstimme und wenigen Enthaltungen gekürte Hajduk warnte davor, dass man die SPD „in der Regierung nicht alleine lassen“ dürfe, weil Scholz Wirtschaftspolitik auf Kosten der Umwelt betreiben werde. In dasselbe inhaltliche Horn stieß Fraktionschef Jens Kerstan der zudem spekulierte, dass der „kühle Taktiker“ Scholz sich für die „bequemen Linken“ als Koalitionspartner entscheiden könnte. ${ }^{27}$ In beiden Äußerungen zeigte sich, dass die Grünen mit ihrem gewünschten Partner SPD noch fremdelten. Vielleicht hatten beide grünen Spitzenpolitiker zu diesem Zeitpunkt auch schon eine Ahnung davon, dass der Schritt von einer Koalition in die nächste keinesfalls eine solche Selbstverständlichkeit sein würde, wie die Umfragen zu diesem Zeitpunkt noch vermuten ließen.

Im neuen Jahr setzte dann die heiße Phase des Wahlkampfs mit den Wahlparteitagen von CDU, GAL und Linkspartei ein: Der mit 93,5 Prozent der Stimmen zum Spitzenkandidaten der CDU gewählte Ahlhaus attackierte Herausforderer Scholz: „Er verspricht jedem alles. " ${ }^{28}$ Am selben Tag erklärte Ahlhaus im Deutschlandfunk, dass er zu Gesprächen mit der SPD bereit sei. Das war das Dilemma der CDU: Als Regierungspartei musste sie aufgrund der katastrophalen Umfragewerte einen Oppositionswahlkampf führen und die SPD attackieren; gleichzeitig war die Große Koalition mit ebendieser SPD die einzig verbliebene Hoffnung auf eine Regierungsbeteiligung der CDU. Ausdruck dieser Situation war auch die geradezu verzweifelt anmutende Plakatwahlkampagne der CDU, die alle ratlos zurückließ: „Wer Citymaut und neues Schulchaos will, muss Rot-Grün wählen “ lautete eine Botschaft, „Kriminalität minus 25\%. Und nu?“" eine zweite. ${ }^{29}$ In all ihren Aussagen ging die CDU davon aus, dass sie nach den Wahlen nicht mehr regieren würde.

Nur wenig komfortabler waren die Rahmenbedingungen des Wahlkampfs für die Grünen: Zwar konnte die GAL mit der Kritik an einer personell ausgebluteten CDU und deren konservativer Wende unter Ablhaus punkten; zu begründen, warum sie in der Regie-

24 Dazu „Antreten, nicht eintreten“, ebenda, 10. Dezember 2010, S. 9.

25 Siehe „Ahlhaus fordert schärfere Sicherheitsgesetze“, ebenda, 27. Dezember 2010, S. 1.

26 Vgl. „Walter Scheuerl lehnt Wahlkampf mit dem Ex-Bürgermeister ab“, ebenda, 27. Dezember 2010, S. 12; „CDU-Spitze verteidigt Ole von Beust“, ebenda, 28. Dezember 2010, S. 7.

27 Zitiert in: „Hajduk führt GAL in die Wahl“, ebenda, 14. Dezember 2010, S. 8.

28 Zitiert in: „Ahlhaus: Scholz verspricht jedem alles“, faz.net, 9. Januar 2010 (Abruf am 13. Januar 2011).

29 Siehe CDU Hamburg, Die Kampagne - Wahl 2011, http://www.cduhamburg.de (Abruf am 29. Januar 2011). 
rung weiterhin gebraucht wurde, fiel ihr jedoch deutlich schwerer. Die von ihr betriebene Schulreform war im Volksentscheid gescheitert und auf absehbare Zeit politisch beerdigt in einem zehnjährigen „Schulfrieden“, für den Olaf Scholz seine Hand öffentlich ins Feuer gelegt hatte. Auch das zweite große Prestigeprojekt der Grünen in der Koalition, die Stadtbahn, hatte Scholz inzwischen für perspektivlos erklärt. Laut Meinungsumfragen wurde er darin von zwei Dritteln der Hamburger unterstützt. ${ }^{30}$ Spitzenkandidatin Hajduk kritisierte diese Entscheidung des Bürgermeisters in spe scharf. ${ }^{31}$ Dennoch war unverkennbar, dass die GAL von Scholz in die Defensive gedrängt worden war und kein zugkräftiges Wahlkampfthema mehr hatte. GAL-Landeschefin Katharina Fegebank erfand dann auch das bemerkenswerte Argument, dass es starke Grüne in der Regierung brauche, weil „wir vieles verstanden haben, was die letzten zweieinhalb Jahre passiert ist “32. Konsequenz dieser politischen Demut der Grünen war die Tatsache, dass sie für ihr Wahlprogramm nicht einmal eine Überschrift fanden. ${ }^{33}$

Am einfachsten von den drei Parteien hatte es die Linke. Sie wollte nach 2008 erneut in die Bürgerschaft einziehen, stand aber - zumal nach den heftig kritisierten KommunismusÄußerungen ihrer Bundesvorsitzenden Gesine Lötzsch ${ }^{34}$ - in den Wahlumfragen unter Druck. Die Partei suchte ihr Heil in der reinen Lehre und rückte deutlich nach links. In ihrem Wahlprogramm setzte sie sich für alles aus ihrer Sicht sozialpolitisch Wünschenswerte ein: für die Armutsbeseitigung, die Abschaffung von Hartz IV und die Gleichstellung der Geschlechter, für den Neubau von 8.000 Wohnungen jährlich, die kostenfreie Nahverkehrsnutzung und die Rekommunalisierung der Energieversorgung, für den Rückkauf der privatisierten Krankenhäuser, die gebührenfreie Bildung von der Kita bis zur Hochschule und eine umfassende Reichenbesteuerung (Wiedereinführung der Vermögenssteuer, Erhöhung der Erbschaftssteuer und des Spitzensteuersatzes bei der Einkommensteuer), um nur einen kleinen Ausschnitt aus dem Panaroma der Forderungen zu nennen. Darüber hinaus machte die Linke klar, dass aus ihrer Sicht ein Regierungswechsel allein nichts bewirke, sondern nur ein „radikaler Politikwechsel“, was für sie in erster Linie eine Wirtschaftsdemokratie und eine Überwindung der kapitalistischen Verhältnisse bedeutete. Nur mit knapper Mehrheit lehnten die Delegierten es ab, eine etwaige Regierungsteilnahme bereits im Wahlprogramm auszuschließen. ${ }^{35}$

Die siegessichere SPD konnte es sich leisten, als letzte der Rathausparteien in die heiße Wahlkampfphase einzutreten - ein Privileg, das normalerweise Regierungsparteien genießen. Der mit 97,5 Prozent zum Spitzenkandidaten gekürte Scholz hatte die Landesvertreterversammlung am 15. Januar sorgfältig choreographiert. Zwei Tage zuvor hatte er Frank Horch, Präses der Handelskammer und Geschäftsführungsmitglied der Schiffsbaugesell-

30 Vgl. Infratest dimap, HamburgTREND, Dezember 2010, Berlin.

31 Vgl. „Scholz Absage an Stadtbahn löst Proteste aus“, Hamburger Abendblatt, 10. Januar 2011, S. 8.

32 Zitiert in: „Einig nur bei den ersten drei“, ebenda, 10. Januar 2011, S. 8.

33 Vgl. GAL Hamburg, Unser Plan für Hamburg Deine Stadt, Wahlprogramm für die Bürgerschaftswahl 2011, beschlossen auf der Landesmitgliederversammlung am 8. Januar 2011 im Bürgersaal Wandsbek, Hamburg 2011.

34 Siehe Gesine Lötzsch, Wege zum Kommunismus, junge welt, 3. Januar 2011, S. 10.

35 Vgl. Die Linke Hamburg, Für ein soziales Hamburg! Wahlprogramm Bürgerschaftswahl 2001, Hamburg 2011, passim, das Zitat auf S. 3; sowie "Linke geht mit Dora Heyenn in den Wahlkampf“, Hamburger Abendblatt, 10. Januar 2011, S. 9. 
schaft Blohm + Voss, als seinen Kandidaten für den Posten des Wirtschaftssenators präsentiert. Das war ein Schlag ins Kontor der CDU, aber mehr noch der GAL. Ablhaus hatte sich bei seinem Amtsantritt im August 2010 um Horch bemüht, war damit aber bei der GAL auf Widerstand gestoßen. Die Aufnahme von Horch ins Schattenkabinett war vor allem eine Kampfansage gegen den potenziellen Koalitionspartner und demonstrierte, dass Scholz zwar offiziell für Rot-Grün, inoffiziell aber um die absolute Mehrheit kämpfte. Einen Tag vor Beginn des Parteitags zauberte der Spitzenkandidat der SPD mit dem Reeder Erck Rickmers einen zweiten Mann aus der Hamburger Hafenwirtschaft aus dem Ärmel, der als SPD-Neumitglied auf dem sicheren Platz 13 der Landesliste kandidieren sollte. Der Parteitag stand so ganz im Zeichen des Schulterschlusses der SPD mit der Hamburger Wirtschaft. Die SPD konnte es vor Glück kaum fassen, dass sie scheinbar aus dem Nichts wieder zur „Hamburg-Partei“ geworden war. Sie dankte es ihrem Landesvorsitzenden damit, dass sie auf inhaltliche Diskussionen und Kampfkandidaturen verzichtete. In ihrem „Regierungsprogramm“ versprach die SPD, dass Hamburg unter einem „Ersten Bürgermeister Olaf Scholz“ wieder „ordentlich regiert“ würde. „Klarheit. Vernunft. Verantwortung“ waren die drei Schlüsselbegriffe des „guten Regierens“, die die SPD dann im Vorgriff auf das Wahlergebnis mit dem Konterfei des „Ersten Bürgermeisters“ flächendeckend in Hamburg plakatierte. ${ }^{36}$

Auch die Bundesprominenz der Parteien schaltete sich ab Januar selbstverständlich in den Hamburger Wahlkampf ein: FDP-Vorsitzender Guido Westerwelle bot der SPD eine Koalition an; das Angebot schlugen aber sowohl Scholz als auch der SPD-Bundesvorsitzende Sigmar Gabriel aus. Der damals noch ungebrochen populäre CSU-Verteidigungsminister Karl-Theodor zu Guttenberg zog mit großem Pomp, begleitet von der Filmmusik „Fluch der Karibik“, in den großen Saal des Hamburger Congress Centrums CCH ein und begeisterte mit seiner markigen wie inhaltsleeren Rede 2.200 Anhänger. Etwas leisere Töne wählte Bundeskanzlerin Angela Merkel, die sich an ihre Geburt in einem Hamburger Krankenhaus erinnerte und die Hansestadt eine „tolle Stadt“ fand. CDU-Generalsekretär Hermann Gröhe sprang seinen Hamburger Parteifreunden bei, indem er Scholz „mangelnde(n) Respekt gegenüber dem Wähler“ vorhielt, weil er sich schon wie ein gewählter Bürgermeister geriere. Der grüne Fraktionsvorsitzende im Bundestag, Jürgen Trittin, hielt die Elbvertiefung „nicht für notwendig“, musste aber einräumen, dass sie beschlossene Sache sei und von den Grünen in Hamburg nicht mehr verhindert werden könne. FDP-Generalsekretär Christian Lindner forderte, aus Hamburg eine „Bildungsstadt“ zu machen und förderte damit ein Thema, das Spitzenkandidatin Suding besonders am Herzen lag. Die damals noch amtierende Vizepräsidentin des Europäischen Parlaments, Silvana Koch-Mehrin, kam an die Elbe und ließ sich zusammen mit Suding im leuchtend-gelben Friesennerz, dem zentralen Plakatmotiv Sudings, ablichten. „Wenn die Inhalte stimmen, kann ein hübsches Gesicht helfen“, ließ Koch-Mehrin sich in Hamburgs größter Regionalzeitung zitieren. In den letzten Tagen vor der Wahl kamen dann schließlich die Bundesparteivorsitzenden von CDU, SPD und FDP sowie die Fraktionsvorsitzenden der Grünen und der Linken im

36 Zum Ganzen „Handelskammer-Chef tritt für Hamburgs SPD an“, ebenda, 14. Januar 2011, S. 1; „Reeder Erck Rickmers tritt für die SPD an“, ebenda, 15. Januar 2011, S. 8; „SPD feiert Kandidaten aus der Wirtschaft“, ebenda, 17. Januar 2011, S. 1; „Die Genossen feiern die Bosse“, ebenda, S. 10; SPD Hamburg, Unser Hamburg: Stark und solidarisch. SPD-Regierungsprogramm für Hamburg, Hamburg 2011. 
Bundestag zu den traditionellen Abschlusskundgebungen ihrer jeweiligen Partei nach Hamburg. ${ }^{37}$

Weder der CDU noch der GAL gelang es während des gesamten Wahlkampfs, die SPD in die Defensive zu drängen. Ein Thema bot sich durchaus an, wie vor allem die CDU erkannte: der Widerspruch zwischen dem Versprechen der SPD, für eine Konsolidierung des Haushalts zu sorgen und den doch sehr weitreichenden sozialen Wohltaten, die sie zur gleichen Zeit in Aussicht stellte. Für die Abschaffung der Kita- und der Studiengebühren sowie für weitere Vorhaben würde die SPD 200 Millionen Euro im Jahr einsparen müssen. Sie drückte sich lange davor, konkrete Sparvorschläge zu machen. Als sie es dann doch tat, brandmarkten Ahlhaus und CDU-Sozialsenator Dietrich Wersich diese als unseriös. Die Glaubwürdigkeit der CDU-Finanzpolitik war jedoch selbst nicht über alle Zweifel erhaben: Während die Christdemokraten in ihrem Wahlprogramm eine Einhaltung des Schuldenverbots ab 2013 in Aussicht stellten ${ }^{38}$, ging der von ihnen geführte Senat in seiner Finanzplanung noch von einer Neuverschuldung von 680 Millionen Euro aus. Das machte es SPD-Finanzexperte Peter Tschentscher leicht, die CDU-Kritik als „durchsichtiges politisches PR-Manöver" zu entlarven. Insgesamt gelang es den Christdemokraten nicht, aus dem Thema Kapital zu schlagen, was wohl auch damit zusammenhing, dass die Medien und die Hafenwirtschaft in Hamburg bemerkenswert unverhohlen auf einen Bürgermeister Scholz setzten. Das zeigte sich auch drei Wochen vor der Wahl in dem ungewöhnlichen Vorgang, dass der Unternehmensverband Hafen Hamburg den Hafenentwicklungsplan des geschäftsführenden CDU-Senats in beispielloser Schärfe öffentlich kritisierte. ${ }^{39}$

So überraschte es am Ende des Wahlkampfs kaum, dass sich das Umfragebild eine Woche vor der Wahl gegenüber der Ausgangslage im Dezember 2010 fast gar nicht verändert, eher noch leicht zugunsten der SPD verbessert hatte: Noch immer waren sieben von zehn Wählern unzufrieden mit der Arbeit des Senats; noch immer äußerten zwei Drittel der Hamburger einen Wechselwunsch. In der „Direktwahlfrage“ zum bevorzugten Bürgermeister distanzierte Herausforderer Scholz den amtierenden Bürgermeister nach wie vor um mehr als vierzig Prozentpunkte. Die SPD hatte der CDU wie schon im Dezember auf allen Kompetenzfeldern mit Ausnahme der Kriminalitätsbekämpfung den Rang abgelaufen, und immer noch wünschten sich die meisten, nämlich 49 Prozent der Befragten, eine rot-grüne Koalition. Eine Alleinregierung der SPD bewerteten inzwischen immerhin 44 Prozent po-

37 Vgl. der Reihe nach „Westerwelle offen für Rot-Gelb in Hamburg“, Hamburger Abendblatt, 14. Januar 2011, S. 1, S. 4; „SPD-Chef Gabriel: Nicht mit Hamburgs FDP“, ebenda, 22. Januar 2011, S. 1, S. 4; „Wahlkampf auf großer und kleiner Bühne“, ebenda, 18. Januar 2011, S. 8; „Merkel macht Ahlhaus Mut“, ebenda, 24. Januar 2011, S. 8; „Scholz tut so, als sei alles entschieden“, ebenda, 3. Februar 2011, S. 4; „Ich halte die Elbvertiefung nicht für notwendig“, ebenda, 7. Februar 2011, S. 4; „Hamburg muss Bildungsstadt werden“ und „Suding bekommt Wahlkampfhilfe aus Brüssel“, ebenda, 11. Februar 2011, S. 4; „Gysi betont Bedeutung der Bürgerschaftswahl“, ebenda, 17. Februar 2011, 12; Schützenhilfe aus Berlin“, ebenda, 18. Februar 2011, S. 8.

38 Siehe CDU Hamburg, Wahlprogramm 2011, Hamburg 2011, S. 10.

39 Zum Ganzen auch hier der Reihe nach „Ahlhaus bittet zur Großen Koalition“, Hamburger Abendblatt, 20. Januar 2011, S. 10; „Ahlhaus wirft Scholz Wahlbetrug vor“, ebenda, 27. Januar 2011, S. 10; „Scholz legt Zahlen auf den Tisch“, ebenda, 1. Februar 2011, S. 8; „CDU-Senator wirft Scholz ,Verarsche” vor“, ebenda, 2. Februar 2011, S. 1; „Das ignorierte Schuldenverbot“, ebenda, 28. Januar 2011, S. 8; „Unternehmen kritisieren Hafenpolitik scharf“, ebenda, 3. Februar 2011, S. 25. 
sitiv. Deutlich weniger positiv wurden dagegen die beiden anderen politisch noch möglichen Bündnisse bewertet, die Große (36 Prozent) und die rot-gelbe Koalition (23 Prozent). ${ }^{40}$ Auch beim allseits erwarteten Überwinden der Sperrklausel durch Linke und FDP lief es folglich auf Rot-Grün oder eine Alleinregierung der SPD zu.

\section{Das Wahlergebnis}

Das Ergebnis war dann trotzdem ein Paukenschlag: 48,4 Prozent und 62 Mandate für die SPD waren ein Ergebnis, das an längst vergangene Zeiten der ehemaligen „Hamburg-Partei“ anknüpfte. Zuletzt war es Henning Voscherau 1991 gelungen, die absolute Mehrheit für die Sozialdemokraten zu erringen. Olaf Scholz konnte in Voscheraus Fußstapfen treten, weil die CDU mit 21,9 Prozent ihr schwächstes Ergebnis bei einer Bürgerschaftswahl nach dem Zweiten Weltkrieg erzielte und ihre Fraktion um die Hälfte von 56 auf 28 Mitglieder schrumpfte. Die Grünen blieben mit 11,2 Prozent der Wählerstimmen und 14 Abgeordneten, trotz leichter Zugewinne gegenüber 2008, deutlich unter ihren Erwartungen. Die Linke konnte ihr Ergebnis von 2008 wiederholen und kehrte mit acht Abgeordneten in die Bürgerschaft zurück. Der FDP gelang dank dem einnehmenden Auftritt ihrer fotogenen Spitzenkandidatin der Sprung über die Fünfprozenthürde mit dem besten Ergebnis in Hamburg seit 37 Jahren. Sie stellte neun Abgeordnete in der neuen Bürgerschaft. Nicht ins Parlament schafften es die Piratenpartei, die mit ihren 2,1 Prozent aber immerhin einen Anspruch auf staatliche Parteienfinanzierung erwarb, die NPD (0,9 Prozent) und die Freien Wähler (0,7 Prozent) sowie einige weitere Kleinparteien. Die Wahlbeteiligung sank auf den historischen Tiefstand von 57,3 Prozent - 6,2 Punkte weniger als noch 2008 (vgl. Tabelle 1).

Bei der Bürgerschaftswahl kam ein gegenüber 2008 erneut verändertes Wahlrecht zur Anwendung, das aufgrund seiner Kompliziertheit - neben anderen Faktoren wie dem weitgehend feststehenden Regierungswechsel, der Inhaltsarmut des Wahlkampfs und der Enthaltung enttäuschter CDU-Wähler - zum erneuten Sinken der Wahlbeteiligung beitrug. Nur spekuliert werden kann, ob das Wahlrecht - ein Mehrstimmenwahlrecht mit zweimal fünf Stimmen, die auf den freien Listen beliebig kumuliert und panaschiert werden können - auch die Größenrelationen zwischen den Parteien beeinflusste. Die Erwartung der Wahlrechtsinitiatoren von „Mehr Demokratie“ war, dass die erweiterten Optionen eher die kleinen Parteien begünstigen würden. Es konnte auch tatsächlich statistisch ermittelt werden, dass die drei kleinen Parteien auf den Stimmzetteln parteiübergreifend panaschierender Wähler in Relation zu ihrem Landesergebnis überdurchschnittlich erfolgreich waren. Insofern profitierten sie scheinbar vom Panaschieren. Auf der anderen Seite wurde von der Möglichkeit der Stimmenverteilung auf der Landesliste nur von etwa 14 Prozent der Wähler Gebrauch gemacht, während 86 Prozent ihre Stimmen auf einen Parteivorschlag bündelten. Unter den Wählern, die ihre Stimmen kumulierten, schnitten die großen Parteien und besonders die SPD besser ab als im Durchschnitt aller Wähler. Dies lässt vermuten, dass unter den spezifischen Kontextbedingungen der Wahl 2011 die Sozialdemokraten vom

40 Vgl. Infratest dimap, WahlREPORT Hamburg 2011, a.a.O. (Fn. 7), S. 17 - 34; Forschungsgruppe Wahlen, Wahl in Hamburg, a.a.O. (Fn. 2), S. 21 - 35. 


\begin{tabular}{|c|c|c|c|c|c|c|c|}
\hline \multicolumn{8}{|c|}{$\begin{array}{l}\text { Tabelle 1: Das Ergebnis der Wabl zur Hamburgischen Bürgerschaft am 20. Februar } 2011 \\
\text { (in Klammern Veränderungen gegenüber 2008) }\end{array}$} \\
\hline & \multicolumn{2}{|c|}{ Landesliste (LL) } & \multicolumn{2}{|c|}{ Wahlkreise (WK) } & \multicolumn{3}{|c|}{ Sitze } \\
\hline & Anzahl & $\%$ & Anzahl & $\%$ & Insgesamt & LL & WK \\
\hline Wahlberechtigte & 1.254 .638 & & 1.254 .638 & & & & \\
\hline Wahlbeteiligung & 718.876 & $57,3 \quad(-6,2)$ & 718.876 & 57,3 & & & \\
\hline Briefwahlquote & 221.456 & $30,8 \quad(+6,6)$ & 221.456 & 30,8 & & & \\
\hline $\begin{array}{l}\text { abgegebene } \\
\text { ungültige } \\
\text { gültige Stimmhefte }\end{array}$ & $\begin{array}{r}717.846 \\
21.278 \\
696.568 \\
\end{array}$ & $\begin{array}{c}(100) \\
3,0 \\
97,0 \\
\end{array}$ & $\begin{array}{r}716.826 \\
26.034 \\
690.792 \\
\end{array}$ & $\begin{array}{r}(100) \\
3,6 \\
96,4 \\
\end{array}$ & & & \\
\hline Gültige Stimmen & 3.444 .602 & $(100)$ & 3.414 .244 & $(100)$ & 121 & 50 & 71 \\
\hline SPD & 1.667 .804 & $48,4(+14,3)$ & 1.547 .133 & 45,3 & $62(+17)$ & 25 & 37 \\
\hline $\mathrm{CDU}$ & 753.805 & $21,9(-20,7)$ & 784.663 & 23,0 & $28(-28)$ & 10 & 18 \\
\hline Grüne/GAL & 384.502 & $11,2 \quad(+1,6)$ & 485.954 & 14,2 & $14(+2)$ & 2 & 12 \\
\hline FDP & 229.125 & $6,7 \quad(+1,9)$ & 214.993 & 6,3 & $9(+9)$ & 8 & 1 \\
\hline Linke & 220.428 & $6,4 \quad( \pm 0,0)$ & 237.634 & 7,0 & $8 \quad( \pm 0)$ & 5 & 3 \\
\hline Piraten $^{1}$ & 73.126 & $2,1 \quad(+1,9)$ & 70.403 & 2,1 & & & \\
\hline $\mathrm{NPD}^{1}$ & 30.648 & $0,9 \quad(+0,9)$ & 35.359 & 1,0 & & & \\
\hline Die Partei ${ }^{1}$ & 23.994 & $0,7 \quad(+0,4)$ & 7.176 & 0,2 & & & \\
\hline Freie Wähler ${ }^{1}$ & 23.135 & $0,7 \quad(+0,7)$ & 16.975 & 0,5 & & & \\
\hline Rentner $^{1}$ & 15.847 & $0,5 \quad(+0,5)$ & 1.531 & 0,0 & & & \\
\hline ÖDP ${ }^{1}$ & 10.464 & $0,3 \quad(+0,2)$ & 3.963 & 0,1 & & & \\
\hline Bürgerliche Mitte ${ }^{2}$ & 8.380 & $0,2 \quad(+0,2)$ & & & & & \\
\hline BIG Hamburg $^{2}$ & 3.344 & $0,1 \quad(+0,1)$ & & & & & \\
\hline Übrige $^{3}$ & & & 8.460 & 0,2 & & & \\
\hline $\begin{array}{l}1 \text { Wahlbewerbungen } \\
2 \text { Wahlbewerbungen } \\
3 \text { Wahlbewerbungen } \\
\text { Quelle: Landeswahla }\end{array}$ & $\begin{array}{l}\text { it Landeslist } \\
\text { ur mit Land } \\
\text { hne Landesli } \\
\text { chuss, Amtl }\end{array}$ & $\begin{array}{l}\text { die nicht in all } \\
\text { liste, ohne Kanc } \\
\text { e, die nur in ein } \\
\text { hes Endergebnis }\end{array}$ & $\begin{array}{l}\text { Wahlkreise } \\
\text { daturen in V } \\
\mathrm{m} \text { der Wahll } \\
\text { Hamburg, } 4\end{array}$ & andidie & ten. & & \\
\hline
\end{tabular}

Wahlrecht profitierten, weil sie durch die Möglichkeit des Kumulierens in den Genuss eines verstärkten „bandwagon“-Effektes kamen. ${ }^{41}$ Diese Vermutung wird dadurch gestützt, dass der SPD von allen Bürgerschaftsparteien das Stimmensplitting am stärksten zugute kam: Ihr Landesstimmenanteil lag 3,1 Prozentpunkte über ihrem Stimmenanteil in den Wahlkreisen, was fast genau dem negativen Saldo der GAL in der Splitting-Bilanz entsprach. Im bürgerlichen Lager profitierte erwartungsgemäß die FDP vom Splitting (vgl. Tabelle 1).

\subsection{Landeslisten- und Wahlkreisergebnisse}

Von der durch das neue Wahlrecht eingeräumten Möglichkeit, auch auf den Landeslisten Personen statt Parteien zu wählen, machten die Hamburger nur mäßig Gebrauch. Insge-

41 Vgl. Patrick Horst, Das neue Hamburger Wahlrecht auf dem Prüfstand: kontraproduktiv, aber schwer reformierbar, in: ZParl, 42. Jg. (2011), H. 4, S. 707 -724, S. 720. 
samt vergaben die Wähler 53 Prozent ihrer Stimmen in traditioneller Art und Weise an die Parteilisten und nur 47 Prozent an Personen. Einzig die Wähler der SPD wählten häufiger spezielle Kandidaten als die Gesamtliste. Dabei ist jedoch zu berücksichtigen, dass die Spitzenkandidaten, allen voran Olaf Scholz für die SPD und Katja Suding für die FDP, das Gros der Personenstimmen auf sich vereinten. ${ }^{42}$ Zieht man die Stimmen der ohnehin sicher gewählten Spitzenkandidaten unter der Prämisse, dass sie faktisch Parteistimmen darstellen, von den Personenstimmen ab, so erhält man ein aussagekräftigeres Bild: Grüne und Linke machten demnach von dem Recht der Persönlichkeitswahl den ausgiebigsten Gebrauch, gefolgt von CDU und FDP. Am wenigsten nutzten die Wähler der SPD die Möglichkeit zur Personenwahl. Insgesamt führte das Verhältnis der Listen- zu den Personenstimmen zu einem sehr ausgewogenen Verhältnis der über die Listen- beziehungsweise Peronenbank besetzten Sitze im Parlament: Von den 50 Landeslistensitzen wurde die eine Hälfte aus dem starren Listensegment, die andere Hälfte über die persönlichen Präferenzstimmen besetzt. Bei CDU, GAL und FDP waren die Sitzkontingente ebenfalls exakt hälftig verteilt; die SPD hatte ein minimales Übergewicht in der Personenbank, die Linke in der Listenbank (vgl. Tabelle 2).

Aus Sicht der Parteien und Fraktionen bedeutete dies, dass die sicheren Plätze auf der Landesliste durchaus spärlich gesät waren: Bei der SPD waren diesmal die ersten zwölf Positionen sicher, bei der CDU aufgrund ihres schlechten Wahlergebnisses gar nur die ersten fünf. Größte Leidtragende des neuen Wahlrechts war die GAL, die paradoxerweise bisher immer seine stärkste Befürworterin gewesen war. Weil sie als drittstärkste Partei in Hamburg in fast allen Wahlkreisen konkurrenzfähig war, konnte sie grundsätzlich mit gar keinem sicheren Landeslistenplatz rechnen - diesmal war es immerhin einer: Position sechs, weil die ersten fünf über Doppelkandidaturen in den Wahlkreisen zum Zuge kamen. Linkspartei (drei) und FDP (vier) erzielten nur wenig mehr sichere Listenplätze (vgl. Tabelle 2).

Vor allem die beiden großen Parteien hatten die Möglichkeit, ihre Kandidaten auf dem ersten Listenplatz in den 17 Mehrpersonenwahlkreisen abzusichern. Wie schon 2008 (bei allerdings weniger flexiblen Listen) wurde auch bei der diesjährigen Bürgerschaftswahl jeder der 17 Wahlkreiskandidaten von SPD und CDU auf Platz eins der Wahlkreisliste gewählt. Erst ab Position zwei wurde es für die Kandidaten der großen Parteien unsicher. Der CDU gelang es diesmal nur in ihrer traditionellen Hochburg (Alstertal - Walddörfer) ein zweites Wahlkreismandat zu erringen. Die SPD dagegen eroberte mit Ausnahme zweier kleiner Dreipersonenwahlkreise in den innerstädtischen Grünen-Hochburgen Rotherbaum und Eimsbüttel in allen Wahlkreisen mindestens zwei Direktmandate, in ihren diesmaligen Hochburgen Bramfeld, Billstedt, Rahlstedt, Bergedorf und auch in Barmbek-Uhlenhorst sogar drei. Linkspartei und FDP hatten nur in den wenigsten Wahlkreisen die Chance, einen Kandidaten durchzubringen. Für die Linkspartei waren drei Kandidaten in ihren Hochburgen Hamburg-Mitte, Billstedt und Altona erfolgreich - jeweils von Wahlkreislistenplatz eins aus. In der liberalen Hochburg Blankenese eroberte Katja Suding für die FDP ein Direktmandat. Bei der GAL reüssierten zwölf Kandidaten in den Wahlkreisen. In ihren Hochburgen Rotherbaum (Till Steffen), Altona (Christa Goetsch) und Eppendorf (Anja Ha$j d u k$ ) kandidierten ihre landespolitischen Zugpferde zur Sicherheit jeweils auf Platz eins. 


\begin{tabular}{|c|c|c|c|}
\hline \multicolumn{4}{|c|}{$\begin{array}{l}\text { Tabelle 2: Die Anzahl der über die Landeslisten gewählten Abgeordneten der Parteien, getrenn } \\
\text { nach Listen- und Personenstimmen }\end{array}$} \\
\hline Alle Parteien & Absolut & Prozent & Sitze \\
\hline Gesamtstimmen & 3.444 .602 & 100 & 50 \\
\hline Listenstimmen & 1.825 .687 & 53,3 & 25 \\
\hline $\begin{array}{l}\text { Personenstimmen } \\
\text { ohne Spitzenkandidaten }\end{array}$ & $\begin{array}{r}1.618 .915 \\
636.550\end{array}$ & $\begin{array}{l}46,7 \\
18,5\end{array}$ & 25 \\
\hline \multicolumn{4}{|l|}{$\mathrm{CDU}$} \\
\hline Gesamtstimmen & 753.805 & 100 & 10 \\
\hline Listenstimmen & 408.774 & 54,2 & 5 \\
\hline $\begin{array}{l}\text { Personenstimmen } \\
\text { ohne Spitzenkandidaten }\end{array}$ & $\begin{array}{l}345.031 \\
150.520\end{array}$ & $\begin{array}{l}45,8 \\
20,0\end{array}$ & 5 \\
\hline \multicolumn{4}{|l|}{ SPD } \\
\hline Gesamtstimmen & 1.667 .804 & 100 & 25 \\
\hline Listenstimmen & 794.606 & 47,6 & 12 \\
\hline $\begin{array}{l}\text { Personenstimmen } \\
\text { ohne Spitzenkandidaten }\end{array}$ & $\begin{array}{l}873.198 \\
240.179\end{array}$ & $\begin{array}{l}52,4 \\
14,4\end{array}$ & 13 \\
\hline \multicolumn{4}{|l|}{ GAL } \\
\hline Gesamtstimmen & 384.502 & 100 & 2 \\
\hline Listenstimmen & 232.388 & 60,4 & 1 \\
\hline $\begin{array}{l}\text { Personenstimmen } \\
\text { ohne Spitzenkandidaten }\end{array}$ & $\begin{array}{l}152.114 \\
110.876 \\
\end{array}$ & $\begin{array}{l}39,6 \\
28,8\end{array}$ & 1 \\
\hline \multicolumn{4}{|l|}{ FDP } \\
\hline Gesamtstimmen & 229.125 & 100 & 8 \\
\hline Listenstimmen & 128.712 & 56,2 & 4 \\
\hline $\begin{array}{l}\text { Personenstimmen } \\
\text { ohne Spitzenkandidaten }\end{array}$ & $\begin{array}{r}100.413 \\
45.012\end{array}$ & $\begin{array}{l}43,8 \\
19,6\end{array}$ & 4 \\
\hline \multicolumn{4}{|l|}{ Linke } \\
\hline Gesamtstimmen & 220.428 & 100 & 5 \\
\hline Listenstimmen & 132.435 & 60,1 & 3 \\
\hline $\begin{array}{l}\text { Personenstimmen } \\
\text { ohne Spitzenkandidaten }\end{array}$ & $\begin{array}{l}87.993 \\
63.009\end{array}$ & $\begin{array}{l}39,9 \\
28,6\end{array}$ & 2 \\
\hline
\end{tabular}

GAL-Fraktionschef Jens Kerstan ging in Bergedorf ein höheres Risiko ein, wurde im dortigen Fünfpersonenwahlkreis aber auch erneut gewählt. ${ }^{43}$

Die GAL war die einzige Partei, bei der sich die Wähler gegen zwei Wahlkreisspitzenkandidaten aussprachen und die auf Listenplatz zwei positionierten Kandidaten bevorzugten. Ansonsten kam es nur bei der SPD zu zahlreichen Kapriolen in den Wahlkreisen: Sieben (knapp ein Fünftel) der direkt gewählten Kandidaten der SPD zogen von Plätzen in die Bürgerschaft ein, die bei starren Listen nicht zum Zuge gekommen wären. Insgesamt revidierten die Wähler die Wahlkreislisten der Parteien jedoch weniger umfangreich als die

43 Vgl. Statistikamt Nord, Wahlen zur Bürgerschaft und zu den Bezirksversammlungen am 20. Februar 2011. Band 1: Analyse, Hamburg, Juni 2011, S. 12 - 15; sowie Landeswahlausschuss, Amtliches Endergebnis, Hamburg, 4. März 2011. 


\begin{tabular}{|c|c|c|c|c|c|c|c|c|c|}
\hline \multirow[t]{3}{*}{ Tabelle 3} & \multicolumn{9}{|c|}{$\begin{array}{l}\text { Anzahl und Anteil der von hinteren Plätzen der Landes- und Wablkreislisten der Partei- } \\
\text { en in die Bürgerschaft gewählten Abgeordneten }\end{array}$} \\
\hline & \multicolumn{3}{|c|}{ Über Landesliste gewählte } & \multicolumn{3}{|c|}{ Über Wahlkreisliste gewählte } & \multicolumn{3}{|c|}{ Insgesamt gewählte } \\
\hline & $\begin{array}{l}\text { Abge- } \\
\text { ordnete }\end{array}$ & $\begin{array}{l}\text { Hoch- } \\
\text { rücker }\end{array}$ & Prozent & $\begin{array}{l}\text { Abge- } \\
\text { ordnete }\end{array}$ & $\begin{array}{l}\text { Hoch- } \\
\text { rücker }\end{array}$ & Prozent & $\begin{array}{l}\text { Abge- } \\
\text { ordnete }\end{array}$ & $\begin{array}{l}\text { Hoch- } \\
\text { rücker }\end{array}$ & Prozent \\
\hline SPD & 25 & 7 & 28,0 & 37 & 7 & 18,9 & 62 & 14 & 22,6 \\
\hline $\mathrm{CDU}$ & 10 & 3 & 33,3 & 18 & - & - & 28 & 3 & 10,7 \\
\hline GAL & 2 & 1 & 50,0 & 12 & 2 & 16,7 & 14 & 3 & 21,4 \\
\hline FDP & 8 & 1 & 12,5 & 1 & - & - & 9 & 1 & 11,1 \\
\hline Linke & 5 & 2 & 40,0 & 3 & - & - & 8 & 2 & 25,0 \\
\hline Alle & 50 & 14 & 28,0 & 71 & 9 & 12,7 & 121 & 23 & 19,0 \\
\hline
\end{tabular}

Landeslisten: Während sie von den hinteren Plätzen der Landeslisten immerhin 14 Kandidaten in die Bürgerschaft wählten, waren es nur neun, die von hinteren Plätzen auf den Wahlkreislisten auf ein Mandat vorrückten. In der Summe wurden damit aber immerhin 23 von 121 Abgeordneten, also knapp ein Fünftel, von hinteren Listenplätzen in die Bürgerschaft gewählt und verdankten ihren Erfolg dem neuen Wahlrecht (vgl. Tabelle 3). Die Fraktionen, die sich im Fall von SPD, GAL und Linkspartei bis zu einem Viertel aus unerwarteten „Hochrückern“ zusammensetzten, wurden vor nicht geringe Integrationsprobleme gestellt. ${ }^{44}$

\subsection{Wahlergebnisse in sozialstruktureller Perspektive}

In sozialstruktureller Hinsicht war zu erwarten, dass die Wahlbeteiligung mit zunehmendem Alter höher ausfiel - am geringsten war sie mit 44 Prozent in der Altersgruppe der 18- bis 24-Jährigen, am höchsten mit 67 Prozent bei den über 60-Jährigen. Von anderen Wahlen bekannt war auch die Tatsache, dass gerade in diesen beiden Altersgruppen die Frauen deutlich seltener wählen als die Männer, während in den mittleren Altersgruppen die Frauen gegenüber den Männern aufgeholt oder sie sogar schon überholt haben. Bei der Bürgerschaftswahl 2011 beteiligten sich die 25- bis 34-jährigen und die 45- bis 49-jährigen Frauen aktiver an der Wahl als die Männer. Eine Überraschung gab es jedoch bei der Wahlbeteiligung insofern, als laut repräsentativer Wahlstatistik ausgerechnet die mittleren Altersgruppen deutlich seltener zur Wahl gingen als noch 2008. Der (in der repräsentativen Wahlstatistik allerdings nicht ausgewiesene) Rückgang der Wahlbeteiligung von 2008 auf 2011 ging allein auf die 25- bis 59-jährigen Wähler zurück, während die jüngsten und die ältesten Wähler ihre Partizipation gegenüber 2008 steigerten. ${ }^{45}$ Bei den ältesten Wählern war das auch deshalb überraschend, weil sie dem neuen Wahlrecht am skeptischsten gegenüberstanden und auch am häufigsten ungültig wählten. ${ }^{46}$ Wenn man der repräsentativen Wahlstatistik glauben darf, schlug sich diese Skepsis nicht in einer verringerten Wahlbeteiligung nieder.

44 Dieses Problem wird eingehend diskutiert bei Patrick Horst, a.a.O. (Fn. 41).

45 Vgl. Statistikamt Nord, a.a.O. (Fn. 43), S. $38-41$.

46 Dazu schon Patrick Horst, a.a.O. (Fn. 41), S. 721 f. 
Auch bei den Stimmenanteilen der Parteien mischte sich Altbekanntes mit Neuem. Bekannt war, dass die CDU in Hamburg weit überproportional von der Altersgruppe der über 60-Jährigen gewählt wird. Hier erzielte die CDU 2011 einen Stimmenanteil von 32,2 Prozent, während sie in allen Altersgruppen unter 60 Jahren deutlich unter 20 Prozent landete. Auch die FDP wurde überproportional von den über 60-Jährigen - und hier insbesondere den Männern - gewählt; sie hatte aber zudem einen Schwerpunkt in der Altersgruppe der 35- bis 44-Jährigen. Am ausgewogensten war die Altersverteilung unter den Wählern der SPD, während die Grünen nach wie vor die jüngste Wählerschaft anzogen. Sie stellten in der Altersverteilung ihrer Wähler quasi das Gegenstück zur CDU dar: In den Altersgruppen der unter 60-Jährigen erzielte die GAL zwischen 13 und 17 Prozent der Stimmen, bei den über 60-Jährigen gerade einmal 3,5 Prozent. Die Linke fand ebenfalls bei den unter 60-jährigen Wählern überproportionalen Zuspruch; überraschenderweise konnte sie insbesondere bei den jüngsten Wählern - und hier vor allem bei den Frauen - deutlich zulegen. Die etablierten Parteien CDU, SPD und GAL wurden überproportional von Frauen gewählt, Linke und FDP dagegen überdurchschnittlich von Männern. Bei der CDU war der Wählerüberhang beim weiblichen Geschlecht aber allein auf die besonders zahlreichen und überdurchschnittlich partizipationsfreudigen Frauen im Alter von über 60 Jahren zurückzuführen. Sie allein machten 53 Prozent der gesamten weiblichen Wählerschaft der CDU aus. Die über 60-Jährigen beiderlei Geschlechts stellten immer noch die Hälfte der gesamten CDU-Wählerschaft; sie kehrten der CDU gegenüber 2008 jedoch auch am stärksten den Rücken. Gleichzeitig legte die SPD genau in dieser Altersgruppe wahlentscheidend - am stärksten zu. ${ }^{47}$

Der Einfluss des sozialen Status' auf das Wählerverhalten folgte auch 2011 vertrauten Wegen. CDU und FDP erzielten in statushohen Stadtteilen, definiert als solche mit hohem Einkommensniveau und geringem Anteil an Sozialhilfeempfängern, ihre besten Ergebnisse. Während die FDP in diesen Stadtteilen gegenüber 2008 zulegte, verlor die CDU gerade hier überproportional. SPD und Linke waren demgegenüber die beiden Parteien, die in den statusniedrigen Stadtteilen überproportional reüssierten - bei der Linken ging die Schere jedoch erwartungsgemäß deutlich weiter auf. Die SPD dagegen schnitt auch in statushohen Gebieten mit rund 43 Prozent sehr ordentlich ab, hier verzeichnete sie stärkere Zuwächse gegenüber 2008 als in ihren Hochburgen mit niedrigem Sozialstatus. Die GAL war 2011 wie schon vor drei Jahren die einzige „klassenlose“ Partei in Hamburg: Bei ihr spielten die Unterschiede der Wähler im sozialen Status keinerlei Rolle. Das bedenklichste Wahlergebnis unter sozialen Gesichtspunkten war der wie schon 2008 zu beobachtende stärkere Rückgang der Wahlbeteiligung in sozial schwächeren Stadtteilen, auch wenn die Differenz zu den sozial besser gestellten lange nicht mehr so stark ausgeprägt war wie noch 2008. Bestehen blieb aber die problematische Tatsache, dass die Wahlbeteiligung dort, wo der Anteil an Sozialhilfeempfängern hoch ist, im Durchschnitt fast dreißig Prozentpunkte niedriger war als in Stadtteilen mit niedrigem Anteil. ${ }^{48}$

47 Vgl. Statistikamt Nord, a.a.O. (Fn. 43), S. 40 - 44.

48 Nämlich 45,5 gegenüber 72 Prozent. Siehe ebenda, S. 26 - 28. Zur niedrigeren Wahlbeteiligung in sozial schwächeren Stadtteilen eingehend auch Cord Jakobeit / Philipp Hiller / Nils Thomsen / Louisa Frölich, Evaluation des Hamburger Wahlrechts bei der Bürgerschaftswahl 2008, Hamburg, Juni 2009, S. 25 - 33; Patrick Horst, a.a.O. (Fn. 41), S. 721; Cord Jakobeit / Philipp Hiller / Nils Thomsen, Evaluation des neuen Hamburger Wahlrechts bei der Bürgerschaftswahl 2011. Ergebnisse einer Wähler- und Nichtwählerbefragung, Hamburg, Juni 2011, S. 46 - 63. 
Für Nicht-Hansestädter, die nur die Großstadtmetropole wahrnehmen, vielleicht überraschend, zeigten sich auch 2011 wieder die traditionellen Restbestände des Stadt-Land-Cleavages im Bundesland Hamburg: Vor allem die CDU, aber auch die FDP schnitten in dünn besiedelten, ländlich strukturierten Stadtgebieten wie schon 2008 überdurchschnittlich gut ab. Die SPD war dagegen in stark verdichteten, urban besiedelten Stadtteilen leicht im Vorteil, gewann diesmal jedoch in den ländlich geprägten Gebieten äußerst stark hinzu und ereichte auch hier 45 Prozent der Stimmen. Parteien mit eindeutig städtischem Rückhalt waren wie schon 2008 die GAL und die Linkspartei: Ihr Anteil in den urbanen Stadtteilen war doppelt so hoch wie in den ländlichen. ${ }^{49}$

\subsection{Wahlmotive und Wahlthemen}

Bei der diesjährigen Wahlentscheidung standen die landespolitischen Motive wie zuletzt immer im Vordergrund, diesmal sogar so ausgeprägt wie nie zuvor: Für 82 Prozent der Bürger waren landespolitische Themen wichtiger als bundespolitische, nur für 16 Prozent war es andersherum. ${ }^{50}$ Die Bürgerschaftswahl 2011 war in erster Linie eine Abwahl von Schwarz-Grün, wobei die CDU deutlich härter abgestraft wurde als die GAL. 76 Prozent der Hamburger betrachteten die CDU am Ende als nicht mehr regierungsfähig, sieben von zehn zeigten sich mit der Regierungsbilanz von Schwarz-Grün unzufrieden, genauso viele sahen in Ahlhaus den falschen Kandidaten für die CDU ${ }^{51}$, und 64 Prozent fanden, dass Ole von Beust mit seinem Rücktritt im Sommer 2010 Hamburg im Stich gelassen hatte. ${ }^{52}$ Gegen die tief verankerte Überzeugung der Hamburger, dass die CDU reif für die Abwahl war, konnte auch die nach ihrer überwiegenden Einschätzung gute wirtschaftliche Lage der Hansestadt nichts ausrichten. Angst vor Arbeitslosigkeit oder wirtschaftliche Zukunftssorgen spielten in diesem Wahlkampf keine Rolle, wichtigste Themen waren - in dieser Reihenfolge - Schul- und Bildungspolitik, Finanzlage der Stadt, Familienpolitik (Kita-Gebühren) und Verkehrsprobleme. Auf all diesen wahlrelevanten Politikfeldern wie auch in der generellen Kompetenz, die Zukunftsprobleme der Stadt zu lösen, wurde die SPD fähiger eingeschätzt als die CDU. ${ }^{53}$ Die den Parteien zugeschriebene Lösungskompetenz hinsichtlich der inhaltlichen Themen war diesmal für die Hälfte aller Wähler der stärkste Faktor für die Wahlentscheidung, für jeweils ein Viertel waren es die Kandidaten oder die Parteibindung. Die hohe Überlegenheit des SPD-Bürgermeisterkandidaten Scholz im Vergleich zu Ahlhaus gab aber immerhin für ein Drittel der SPD-Wähler den Ausschlag. ${ }^{54}$ Insgesamt begünstigte die Gewichtung der Wahlmotive die Wählervolatilität, die mit 22,7 Punkten nahe an die Rekordwerte von 2004 und 2001 herankam. ${ }^{55}$

49 Vgl. Statistikamt Nord, a.a.O. (Fn. 43), S. 29 - 30.

50 Vgl. Forschungsgruppe Wahlen, a.a.O. (Fn. 2), S. 22.

51 Vgl. Infratest dimap, a.a.O. (Fn. 7), S. 22, S. 25.

52 Vgl. Forschungsgruppe Wahlen, a.a.O. (Fn. 2), S. 22.

53 Vgl. ebenda, S. $30-38$.

54 Vgl. Infratest dimap, a.a.O. (Fn. 7), S. 40.

55 Gemessen als kumulierte Stimmengewinne aller Parteien, die gegenüber 2008 hinzugewannen. Vgl. zu den Werten bei früheren Bürgerschaftswahlen Tabelle 3 in Patrick Horst, Das Parteiensystem Hamburgs, in: Uwe Jun / Melanie Haas / Oskar Niedermayer (Hrsg.), Parteien und Parteiensysteme in den deutschen Ländern, Wiesbaden 2008, S. 224. 


\section{Regierungsbildung und Formierung der Opposition}

Mit der absoluten Mehrheit für die SPD am 20. Februar 2011 war ein ganz entscheidender Parameter für die Regierungsbildung gesetzt: Olaf Scholz musste keine - möglicherweise langwierigen - Koalitionsverhandlungen mit den Grünen führen, sondern konnte sich frei von Koalitionszwängen den Sondierungen über seinen künftigen Senat widmen. Auch seine Partei, die nach einem Jahrzehnt im Niemandsland der Opposition ihren Retter auf Händen trug, störte ihn dabei nicht. Schon auf dem Wahlparteitag im Januar hatte ein Parteitagsdelegierter ironisch über Scholz gemeint: „Er kann jetzt sogar übers Wasser gehen. "56 Das setzte sich in der Regierungsbildung fort, als die Anwärter für einen Senatsposten beim Parteivorsitzenden im Kurt-Schumacher-Haus ihre Aufwartung machten, dieser sich aber bis zum Schluss nicht in die Karten schauen ließ. Nur zwei Senatoren - Wirtschaftssenator Horch und die aus Berlin für das Amt der Kultursenatorin importierte Barbara Kissler - standen vor der Wahl des Ersten Bürgermeisters am 7. März fest. Sofern eine Disziplinierung der SPD-Abgeordneten noch vonnöten war, funktionierte sie, denn Scholz wurde mit 62 Stimmen im ersten Wahlgang gewählt. Weil ein Abgeordneter der SPD fehlte, hatte ihm auch mindestens ein Mitglied der Opposition seine Stimme gegeben. Scholz nahm - ein verfassungsrechtliches Novum in der Hansestadt - für zwei Wochen allein auf der Senatsbank Platz. Ein aussagekräftigeres Symbol für seine Alleinherrschaft in der Partei, die nun das erste Mal in ihrer stolzen Geschichte über einen Parteivorsitzenden im Bürgermeisteramt verfügte, konnte es nicht geben. ${ }^{57}$

Zusammen mit seinem Vertrauten Christoph Krupp, der die zentrale Schaltstelle des Chefs der Senatskanzlei besetzte, machte sich Scholz in den folgenden zehn Tagen an den Neuzuschnitt der Behörden. Erklärtes Ziel war deren Verkleinerung, besonders im Visier des Bürgermeisters standen die beiden Mammutbehörden für Soziales, Familie, Gesundheit und Verbraucherschutz sowie für Stadtentwicklung, Umwelt, Bau und Verkehr. Am 17. März konnte Scholz dann das Ergebnis präsentieren: Der Verkehrsbereich wurde aus der Umweltbehörde herausgelöst und der Behörde für Wirtschaft, Verkehr und Innovation von Frank Horch zugeschlagen. Die Wirtschaftsbehörde musste dafür die Zuständigkeit für die Arbeitsmarktpolitik abgeben. Diese ging an die neu zusammengesetzte Arbeits- und Sozialbehörde unter Detlef Scheele, der künftig auch für Familie und Integration verantwortlich zeichnete. Der 54-jährige Scheele, der seine berufliche Karriere 1985 als persönlicher Referent des damaligen SPD-Landesvorsitzenden Ortwin Runde gestartet und es 2008 unter Scholz zum Staatssekretär im Bundesarbeitsministerium gebracht hatte, wurde damit zum eigentlichen „Supersenator" der neuen Regierung. Die Berufung des ausgewiesenen Arbeitsmarktexperten war ein klares Signal des Bürgermeisters an die Gewerkschaften und in die eigene Partei hinein, dass die Arbeitsmarktpolitik im neuen Senat nicht nur eine Fußnote zur Wirtschaftspolitik sein würde. Horch musste auch noch die zukunftsträchtigen

56 Zitiert bei „Reeder Erck Rickmers tritt für die SPD an“, Hamburger Abendblatt, 15. Januar 2011, S. 8.

57 Vgl. „Olaf Scholz wird wahrscheinlich am 7. März Bürgermeister“, ebenda, 26. Februar 2011, S. 8; "Garantiert nicht handzahm“, ebenda, 28. Februar 2011, S. 17; „Olaf Scholz allein im Senat“, ebenda, 2. März 2011, S. 10; „Das System Scholz oder das Prinzip abgestuften Vertrauens“, ebenda, 5. März 2011, S. 8; „Olaf Scholz ist am Ziel“, ebenda, 8. März 2001, S. 1 und „Junge Mutter sichert Olaf Scholz die Wahl“, ebenda, S. 8. 


\begin{tabular}{|l|l|}
\hline Tabelle 4: Der neue Hamburger Senat 2011 \\
\hline Erster Bürgermeister und Präsident des Senats & Olaf Scholz (SPD), 52 Jahre \\
\hline $\begin{array}{l}\text { Senatorin für Wissenschaft und Forschung (Zweite Bürger- } \\
\text { meisterin) }\end{array}$ & $\begin{array}{l}\text { Dr. Dorothee Stapelfeldt (SPD), } \\
54 \text { Jahre }\end{array}$ \\
\hline Senator für Arbeit, Soziales, Familie und Integration & Detlef Scheele (SPD), 54 Jahre \\
\hline Senatorin für Gesundheit und Verbraucherschutz & $\begin{array}{l}\text { Cornelia Prüfer-Storcks (SPD), } \\
54 \text { Jahre }\end{array}$ \\
\hline Senator für Inneres und Sport & Michael Neumann (SPD), 41 Jahre \\
\hline Senator für Schule und Berufsbildung & Ties Rabe (SPD), 50 Jahre \\
\hline Senatorin für Stadtentwicklung und Umwelt & Jutta Blankau (SPD), 56 Jahre \\
\hline Senator für Wirtschaft, Verkehr und Innovation & Frank Horch (parteilos), 63 Jahre \\
\hline Senator für Finanzen und Bezirksangelegenheiten & Dr. Peter Tschentscher (SPD), 45 Jahre \\
\hline Senatorin für Justiz und Gleichstellung & Jana Schiedek (SPD), 36 Jahre \\
\hline Senatorin für Kultur & Barbara Kisseler (parteilos), 61 Jahre \\
\hline Quelle: http://www.hamburg.de/senat/1959818/hamburger-senat.html (Abruf am 9. Juli 2011). \\
\hline
\end{tabular}

Bereiche Medien und IT-Wirtschaft an die Senatskanzlei abtreten, weshalb CDU-Oppositionschef Dietrich Wersich spotten konnte, wo denn da die Innovation bliebe. Für die verkleinerte Behörde für Gesundheit und Verbraucherschutz gewann Scholz mit Cornelia Prüfer-Storcks, ehemalige Gesundheitsstaatssekretärin in Nordrhein-Westfalen und zuletzt Vorstandsmitglied der AOK Rheinland/Hamburg, zwar immerhin noch eine externe Lösung. Sie war jedoch nur dritte Wahl, nachdem zuvor der ehemalige SPD-Landesvorsitzende Mathias Petersen und die SPD-Bundestagsabgeordnete Carola Reimann abgesagt hatten. Dem neuen Senat gehören elf statt zuvor zehn Mitglieder an, darunter fünf Frauen; auch die Staatsräte vermehrte Scholz um einen auf 15 bei nur zwei Frauen. Glanz versprühte der Senat kaum, sein Hamburger Lokalkolorit war stärker ausgeprägt, als der Bürgermeister zu hoffen gewagt hatte (vgl. Tabelle 4). ${ }^{58}$

Mit der Bestätigung des Senats am 23. März und der anschließenden Regierungserklärung des Bürgermeisters war die Regierungsbildung nach 31 Tagen formal abgeschlossen sie hatte zwei Wochen länger gedauert als beim bisherigen Rekord von Ole von Beust im Jahre 2004.59 Daran zeigte sich, dass zum „guten Regieren“ auch die Maxime „Gründlichkeit vor Schnelligkeit“ gehören sollte. Erst im Sommer wollte Scholz die vorläufige Haushaltsführung beenden und einen regulären Etat aufstellen, der dann auch etwas über die Finanzierung seiner Wahlkampfversprechen verlautbaren würde. Von diesen machte er keine Abstriche: Die Abschaffung der Kita- wie der Studiengebühren, der Bau von 6.000 neuen Wohnungen im Jahr, ein Drittel davon öffentlich gefördert, die Elbvertiefung, die

58 Vgl. der Reihe nach „Ein ,CdS’ aus Bergedorf“, ebenda, 3. März 2011, S. 10; „Scholz will kleine Behörden“, ebenda, 11. März 2011, S. 8; „Stapelfeldt wird Hamburgs Zweite Bürgermeisterin“, ebenda, 18. März 2011, S. 1, „Ein Hauch von Basta“, ebenda, S. 8; http://www.hamburg.de/senatoren/ (Abruf am 29. Juli 2011); Hamburgische Bürgerschaft, Plenarprotokoll 20/2 am 23. März 2011, S. 43; „Das sind die Staatsräte“, Hamburger Abendblatt, 19. März 2011, S. 9; Claus Strunz, Leitartikel: In der Proporzfalle, ebenda, 18. März 2011, S. 2.

59 Vgl. Patrick Horst, Neuwahl 2004, a.a.O. (Fn. 1), S. 267 - 268. 
Hafenquerspange, „das modernste Bussystem Europas“, eine neue S-Bahn und eine Beteiligung der Stadt an den Energienetzen sollten ebenso kommen, wie die Stadtbahn und eine City-Maut verhindert sowie der Schulfrieden gewahrt würden. ${ }^{60}$ Der mediengerecht oft etwas martialisch inszenierte Führungsstil des neuen Bürgermeisters - „Wer bei mir Führung bestellt, bekommt sie auch" ${ }^{61}$ - ließ sich durchaus mit einem großzügigen Entgegenkommen gegenüber der eigenen politischen Klientel und dem empirischen Volkswillen vereinbaren. Scholz hatte, wie er auch schon als Arbeitsminister im ersten Kabinett von Angela Merkel unter Beweis gestellt hatte, aus seiner Zeit als SPD-Generalsekretär gelernt, als er mit der Agenda-Politik des damaligen Bundeskanzlers Gerhard Schröder die Partei gegen sich aufbrachte. Mittlerweile führte er Senat, Partei und Fraktion keineswegs mehr autokratisch, sondern inklusiv, wie sich auch an der gemeinsamen Entwicklung des Arbeitsprogramms des Senats durch den SPD-Landesvorstand, die Fraktion und den Senat zeigte, das am 23. Mai der Öffentlichkeit vorgestellt wurde. ${ }^{62}$

Währenddessen schlugen die ehemaligen Regierungsparteien CDU und GAL unterschiedliche Wege in die Opposition ein: Bürgermeister Ahlhaus sowie Partei- und Fraktionschef Schira hätten zwar gern auch in Zukunft Führungsfunktionen übernommen, mussten aber schnell erkennen, dass sie in Partei und Fraktion nicht mehr an exponierter Stelle erwünscht waren. Schira bemerkte dies eher als Ablhaus, weshalb er von der Fraktion immerhin noch zum stellvertretenden Präsidenten der Bürgerschaft nominiert wurde. Ahlhaus blieb dagegen einfacher Abgeordneter ohne herausgehobene Funktion. Neuer Fraktionsvorsitzender wurde Sozialsenator Wersich, neuer Parteichef nach der ersten konsultativen Mitgliederbefragung der Hamburger CDU der Altonaer Bundestagsabgeordnete Marcus Weinberg. Beide zählen eher zum liberalen Flügel der Partei und befürworteten unter Schwarz-Grün die gescheiterte Primarschulreform. Weinberg setzte sich im innerparteilichen Wettbewerb gegen die Blankeneser Rechtsanwältin Karin Prien, eine erklärte Gegnerin der Schulreform, durch und wurde anschließend mit 71 Prozent der Stimmen auf einem CDU-Parteitag gewählt. Der kleine Rechtsruck der Hamburger CDU unter Ahlhaus blieb ein nicht einmal sechs Monate währendes Intermezzo, die Partei kehrte zum Kurs Ole von Beusts zurück. ${ }^{63}$

Anders als die CDU entschied sich die GAL gegen eine personelle Runderneuerung: Spitzenkandidatin Hajduk erklärte drei Tage nach der Wahl ihren Verzicht auf den Fraktionsvorsitz, wurde aber stellvertretende Fraktionschefin. Die Kampfabstimmung in der 14-köpfigen GAL-Fraktion um die Spitzenfunktion entschied der bisherige Amtsinhaber

60 Siehe Hamburgische Bürgerschaft, Plenarprotokoll 20/2 am 23. März 2011, S. 28 - 41.

61 Dieses Zitat ziert so ziemlich jeden Medienartikel über Scholz. Vgl. nur Frank Pergande, Sicheren Schrittes über gut getarnte Gräben, faz.net, 19. Februar 2011 (Abruf am 21. Februar 2011).

62 Vgl. „Hamburg-Klausur der SPD: Schulterschluss von Partei, Fraktion und Senat - Steuermehreinnahmen zur Konsolidierung einsetzen“, Pressemitteilung der SPD vom 22. Mai 2011, spdhamburg.de (Abruf am 30. Juli 2011), sowie „Wir schaffen das moderne Hamburg“, Arbeitsprogramm des Senats, 10. Mai 2011, http://www.hamburg.de/arbeitsprogramm-senat/ (Abruf am 30. Juli 2011).

63 Siehe „CDU-Aufstand gegen die Parteiführung“, Hamburger Abendblatt, 21. Februar 2011, S. 9; „Schira geht - auch Ahlhaus verzichtet, ebenda, 22. Februar 2011, S. 8; „Wie Ahlhaus und Schira ihre Rechnung ohne die Partei machten, ebenda, 26. Februar 2011, S. 8; „Union sucht klaren Kurs“, ebenda, 1. März 2011, S. 8; „CDU wählt Weinberg zum Parteichef“, http://www.ndr.de/ regional/hamburg/cdu217.html, 15. Juni 2011 (Abruf am 30. Juli 2011). 
Jens Kerstan gegen den früheren Justizsenator Till Steffen klar mit elf zu drei Stimmen für sich. Der Parteivorstand blieb im Amt; die Partei verordnete sich aber auf Druck der Mitglieder einen ausführlichen inhaltlichen „Aufarbeitungsprozess“. Der Parteivorstand räumte selbstkritisch ein, dass er „in Sachen Beteiligungskultur, Politikstil und auch in der Wahlkampfführung “ hinter den grünen Ansprüchen zurückgeblieben sei und es klüger gewesen wäre, bei der Schulreform „auch einmal Zugeständnisse zu machen, statt zu viel auf einmal zu wollen“. Die Grünen hätten „eine falsche Wahrnehmung von der Verankerung unserer Ideen in der Gesellschaft“ gehabt, und Schwarz-Grün hätte „immer wieder an den Menschen vorbeiregiert". Anja Hajduk gestand ein, dass die Fortführung der Koalition nach dem Rücktritt von Beusts im August 2010 „ein Irrtum“ gewesen war. ${ }^{64}$

Die beiden anderen Oppositionsparteien in der Hamburgischen Bürgerschaft hatten den geringsten Grund, sich personell zu erneuern: Die in die Bürgerschaft zurückgekehrte Linke bestätigte die bisherige Fraktionsvorsitzende Dora Heyenn im Amt, die neu ins Parlament eingezogene FDP wählte sich Spitzenkandidatin Katja Suding zur Chefin. ${ }^{65}$ Beide sorgten in der zweiten Plenarsitzung der Bürgerschaft für Aufsehen, als sie den Anspruch erhoben, die zur Nachfolgerin von Dorothee Stapelfeldt gewählte Bürgerschaftspräsidentin Carola Veit ganz (die FDP) oder in Teilen (die Linke) unterstützt zu haben. Veit, die sich innerparteilich knapp gegen den überraschenden Herausforderer Mathias Petersen durchgesetzt hatte, erhielt nur 65 Ja-Stimmen, so wenige wie kein Präsident und keine Präsidentin vor ihr. ${ }^{66}$ Dies war ein erster Hinweis darauf, dass auch in der neuen Scholz-SPD die Flügelstreitigkeiten der in Ehren ergrauten „Hamburg-Partei“ keinesfalls überwunden waren.

\section{Zusammenfassung}

(1) Olaf Scholz, die überragende Führungsfigur der Hamburger SPD, führte die vormalige „Hamburg-Partei“ nach einem Jahrzehnt in der Opposition wieder zurück an die Macht. Die SPD gewann gegenüber 2008 14,3 Prozentpunkte hinzu und erzielte 48,4 Prozent der Stimmen. Das reichte für die absolute Mehrheit von 62 der 121 Sitze in der Bürgerschaft. Erstmals seit 1991 stellte die SPD wieder allein die Regierung.

(2) In erster Linie wurde die Hamburger CDU für das Scheitern der schwarz-grünen Koalition verantwortlich gemacht. Bürgermeister Christoph Ahlhaus, der im August 2010 die Nachfolge von Ole von Beust angetreten hatte, vermochte die Hamburger und auch den grünen Koalitionspartner nicht zu überzeugen. Als mit dem Rücktritt seines Finanzsenators das fünfte Senatsmitglied der CDU seit März 2010 die Regierung verlassen hatte, ergriff die GAL die Gelegenheit, die Koalition aufzukündigen. Damit war das Schicksal der CDU für die bevorstehende Neuwahl besiegelt: Hamburgs führende Regierungspartei des letzten Jahrzehnts stürzte um 20,7 Punkte auf nur noch 21,9 Prozent

64 „Anja Hajduk verzichtet auf GAL-Fraktionsvorsitz, Hamburger Abendblatt, 24. Februar 2011, S. 2; „Duell mit Tradition“, ebenda, 28. Februar 2011, S. 8; „Kerstan siegt mit 11:3“, ebenda, 1. März 2011, S. 8; „Schwarz-Grün hat an den Menschen vorbeiregiert“, ebenda, 3. Mai 2011, S. 11. Alle Diskussionsbeiträge wurden im Internet veröffentlicht. Vgl. http://hamburg.gruene. de/partei/aufarbeitungsprozess (Abruf am 21. Juli 2011).

65 „Heyenn führt Linke-Fraktion“, Hamburger Abendblatt, 1. März 2011, S. 8.

66 „Gelbe Karte für Veit, grünes Licht für Senat“, ebenda, 24. März 2011, S. 11. 
ab. Die CDU-Fraktion halbierte sich auf 28 Mitglieder. Das war das schlechteste Ergebnis der Partei seit ihrem Bestehen.

(3) Die Grünen konnten sich zwar gegenüber der vorherigen Bürgerschaftswahl um 1,6 Punkte auf 11,2 Prozent der Wählerstimmen verbessern, blieben aber deutlich unter ihren Erwartungen. Es gelang ihnen nach dem Scheitern ihrer Vorzeigeprojekte Primarschulreform und Stadtbahn nicht, die Hamburger von der Notwendigkeit ihrer erneuten Regierungsbeteiligung zu überzeugen. Mit 14 Mandaten sind sie die zweitgrößte der vier Oppositionsfraktionen.

(4) Drittgrößte Oppositionspartei wurde die FDP, der zuletzt 2001 der Sprung über die Fünfprozenthürde gelungen war. Sie profitierte von dem frischen Gesicht ihrer Spitzenkandidatin und den enttäuschten CDU-Wählern, die bei ihr Zuflucht suchten. Die FDP erreichte 6,7 Prozent der Stimmen und neun Mandate. Zur vierten Oppositionsfraktion machten die Hamburger die Linkspartei. Sie erzielte mit 6,7 Prozent das gleiche Ergebnis wie 2008 und kehrte mit acht Abgeordneten ins Parlament zurück.

(5) Die Wählervolatilität, die sich in Hamburg 1993 sprunghaft erhöht hatte und seither bei jeder Wahl zweistellige Raten aufwies, reichte mit einem kumulierten Stimmengewinn von 22,7 Punkten nahe an die Rekordwerte von 2004 und 2001 heran. Als Ergebnis nahm die Fragmentierung im Parteiensystem weiter zu. Seit den 1950er Jahren kam es nach 2001 erst zum zweiten Mal zu einem Fünfparteienparlament in Hamburg, außerdem erzielte die Piratenpartei noch 2,1 Prozent der Stimmen.

(6) Die Wahlbeteiligung sank um 6,2 Punkte auf ein neues Rekordtief von 57,3 Prozent der Wahlberechtigten. Neben dem weitgehend feststehenden Regierungswechsel, der Inhaltsarmut des Wahlkampfs und der Wahlenthaltung enttäuschter CDU-Wähler trug auch das komplizierte neue Wahlrecht, ein Zehnstimmenwahlrecht mit freien Listen, seinen Teil zur Wahlenthaltung bei, vor allem bei Wählern aus sozial schwächeren Stadtteilen. Auch die Anteile ungültiger Stimmzettel und von Briefwählern nahmen als Folge des neuen Wahlrechts zu.

(7) Der neue Senat ist angetreten mit dem Versprechen, Hamburg wieder gut zu regieren und sich dabei mehr auf das Machbare als auf das Wünschenswerte zu konzentrieren. Zum Machbaren zählen für Scholz aber durchaus auch teure Wahlkampfversprechen wie die Abschaffung der Kita- und der Studiengebühren. Den von der Schuldengrenze im Grundgesetz verlangten Haushaltsausgleich will er erst langfristig bis 2020 durchsetzen und nicht auf Kosten der sozialen Gerechtigkeit. Zur gleichen Zeit will er den Hafen und den Ausbau der Verkehrswege wieder ins Zentrum seiner Wirtschaftspolitik stellen. Ob dem Bürgermeister der Spagat zwischen scheinbar gegensätzlichen Positionen gelingt, wird auch davon abhängen, ob sich seine Koalition mit der Hamburger Hafenwirtschaft in Gestalt von Wirtschaftssenator Frank Horch als tragfähig und von Dauer erweist. Mit Detlef Scheele, Jutta Blankau und Cornelia Prüfer-Storcks wurden starke Vertreter gewerkschaftlicher Interessen in den Senat eingebunden, die Horch möglicherweise die Lust an seinem neuen, in den Kompetenzen beschnittenen Amt verleiden könnten. 